See discussions, stats, and author profiles for this publication at: https://www.researchgate.net/publication/331431913

\title{
An evaluation of predictors of dropout from an Emotional Coping Skills programme in a community mental health service
}

Article in The Cognitive Behaviour Therapist · July 2019

DOI: $10.1017 /$ S1754470X19000151

CITATIONS

0

2 authors:

Shalini Raman

University of Southampton

5 PUBLICATIONS 1 CITATION

SEE PROFILE
READS

92

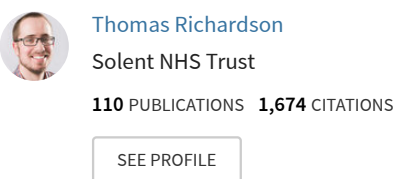

Some of the authors of this publication are also working on these related projects:

Finances in Bipolar View project

Home environment: Association with hyperactivity/impulsivity in children with ADHD and their non-ADHD siblings View project 


\section{Cambridge Core Share}

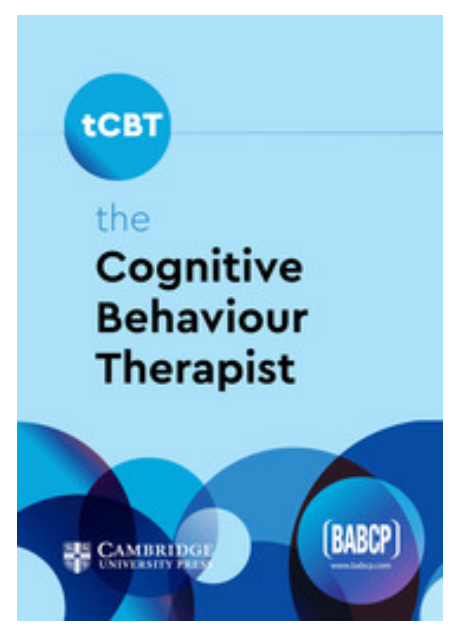

\section{An evaluation of predictors of dropout from an Emotional Coping Skills programme in a community mental health service}

The Cognitive Behaviour Therapist, Volume 12

SHALINI RAMAN, THOMAS RICHARDSON

DOI: $10.1017 / S 1754470 \times 19000151$

Published online: 04 July 2019

Print publication: January 2019

\section{Read this article for free}

\section{Abstract}

Drop-out from mental health services is a significant problem, leading to inefficient use of resources and poorer outcomes for clients. Adapted dialectical behaviour therapy (DBT), often termed Emotional Coping Skills (ECS) programmes, show some of the highest rates of drop-out from therapy recorded in the literature. The present study aimed to add to the evidence base, by evaluating predictors of drop-out from an ECS programme in a UK-based Community Mental Health Team (CMHT). An existing data set of 49 clients, consisting of clients' responses on a number of questionnaires, was evaluated for predictors of drop-out. Predictors of drop-out included symptom severity, substance use and client demographics. Independent-samples $t$-tests and chi-square cross tabs analyses revealed no significant differences between drop-outs and completers of therapy on any of the variables. This suggests that contrary to common assumptions and previous findings, clients using substances, who are highly anxious, or who experience a greater degree of emotion dysregulation, are not more likely to drop out from ECS programmes compared with other individuals. The clinical implications of these findings and future research are discussed within the wider context of the evidence base.

\section{Key learning aims}

(1)To be familiar with common predictors of drop-out from psychological therapies, as indicated by the literature. (2) To understand the theories underlying factors that impact drop-out and the associated consequences for mental health services. (3)To understand the potential impact of staff assumptions of factors that affect drop-out on client retention. (4)To have an understanding of initiatives and strategies that may improve client-retention and engagement in services.

\section{How does Cambridge Core Share work?}

Cambridge Core Share allows authors, readers and institutional subscribers to generate a URL for an online version of a journal article. Anyone who clicks on this link will be able to view a read-only, up-to-date copy of the published journal article. 